\title{
Elimination of refractory ventricular tachycardia storm and fibrillation using stereotactic radiotherapy
}

\author{
Alexander Wutzler ${ }^{1}$, Borris Tiedke ${ }^{1}$, Mohamed Osman ${ }^{1}$, Noha Mahrous ${ }^{1}$, and Reinhard \\ Wurm $^{1}$ \\ ${ }^{1}$ Klinikum Frankfurt Oder GmbH
}

June 11, 2021

\begin{abstract}
Ventricular storms and fibrillation are potentially lethal arrhythmias with limited treatment options. Failed catheter ablation is associated with a fourfold maortality increase in this population. Stereotactic body radiotherapy has been proposed as last resort. We report a patient in whom radiotherapy was safely performed leading to the elimination of arrhythmias

Alexander Wutzler ${ }^{\mathrm{a}, \mathrm{b}}$, Borris Tiedke ${ }^{\mathrm{a}}$, Mohamed Osman ${ }^{\mathrm{a}}$, Noha Mahrous ${ }^{\mathrm{a}}$, Wurm R ${ }^{\mathrm{c}}$

aDepartment of Cardiology, Klinikum Frankfurt (Oder), Germany

bCardiovascular Center, St. Josef Hospital, University Hospital of the Ruhr-University Bochum

cDepartment of Radiation Oncology, Klinikum Frankfurt (Oder), Germany

Corresponding Author:

Mahrous, Noha

Department of Cardiology

Klinikum Frankfurt (Oder), Müllroser Chaussee 7

15236 Frankfurt (Oder)

Email: Noha.Mahrous@klinikumffo.de

Phone: +49 1784188268

Fax: +493355484652

All authors declare no conflict of interest.

Funding: none to declare
\end{abstract}

A.W. and R.W. conceived of the presented idea und developed the theory, and together with M.O. and B.T performed the procedures and the follow up. N.M. wrote the manuscript. All authors discussed the results and contributed to the final manuscript

Key clinical message Radiotherapy offers a feasible, safe, and effective therapy to VT storms and VF refractory to pharmacotherapy and ablation.

Introduction 
Ventricular tachycardia (VT) storms and ventricular fibrillation (VF) are arrhythmias with limited and unsatisfactory treatment options and are associated with high mortality. Failed catheter ablation poses a fourfold increased risk of death in patients with VT/VF. Furthermore, heart failure and adequate implantable cardioverter defibrillator (ICD) shocks are associated with impaired prognosis ${ }^{[1]}$. Stereotactic body radiotherapy (SBRT) has been proposed as a last resort in patients with VT storm and VF refractory to medical therapy and catheter ablation ${ }^{[2]}$.

Case report

A 56-year- old male patient with history of myocardial infarction and heart failure presented to our hospital with cardiac arrest due to VT storm that degenerated to ventricular fibrillation. The VT storm was refractory to pharmacotherapy. The patient experienced a previous episode of VF ten years ago, following which he underwent multiple revascularizations of the left anterior descending (LAD) coronary artery. He had ever since a left ventricular aneurysm with decreased left ventricular ejection fraction (LVEF) (15\%). He underwent ICD implantation seven years ago. ICD Interrogation revealed multiple VT and VF episodes within the last 6 Weeks, which were treated with ATP or adequate ICD shocks. After resuscitation and stabilization, the patient underwent catheter ablation of VT. Electroanatomical mapping revealed an anterior and antero-septal scar area in the left ventricle. Catheter ablation was performed. Nonetheless, the VT recurred after three months. Due to therapy- refractoriness, extensive substrate, and involvement of the septum, SBRT was performed. Cardiac radiotherapy was delivered in a single dose of (25 Gy) high precisionSBRT based on the electroanatomic mapping.

SBRT was performed without complications or ICD dysfunction. During a one- year follow up via ICD telemonitoring as well as outpatient clinic assessments, no recurrence of any VT was documented, after a massive periinterventional VT burden. The LVEF improved to 31\%. No complications related to SBRT were detected. The patient remained on Amiodarone, B-blockers, platelet inhibitor, diuretics and a Sacubitril/valsartan combination.

Conclusion

Radiotherapy offers a feasible, safe, and effective therapy to VT storms and VF refractory to pharmacotherapy and ablation. We report a patient in whom radiotherapy was safely performed leading to complete elimination of ventricular arrhythmia during mid-term follow up. Our results should be confirmed in a prospective multi-center trial.

\section{References}

1- Nayyar S, Ganesan AN, Brooks AG, Sullivan T, Roberts-Thomson KC, Sanders P. Venturing into ventricular arrhythmia storm: a systematic review and meta-analysis. European heart journal. 2013 Feb 21;34(8):56071.

2- Scholz EP, Seidensaal K, Naumann P, André F, Katus HA, Debus J. Risen from the dead: Cardiac stereotactic ablative radiotherapy as last rescue in a patient with refractory ventricular fibrillation storm. HeartRhythm case reports. 2019 Jun 1;5(6):329-32. 


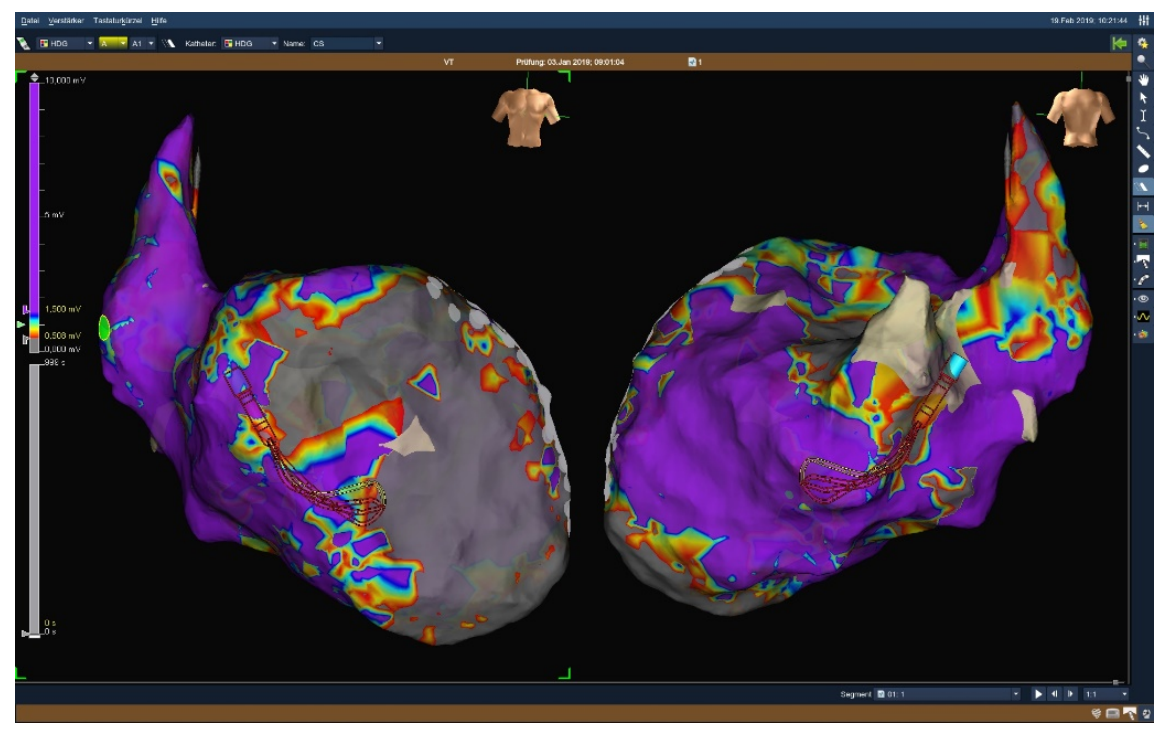

